\title{
ORBITAL PERTURBATIONS OF THE GALILEAN SATELLITES DURING PLANETARY ENCOUNTERS
}

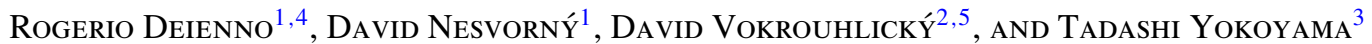 \\ ${ }^{1}$ Southwest Research Institute, Boulder, CO, USA; rogerio.deienno@gmail.com \\ ${ }^{2}$ Institute of Astronomy, Charles University, Prague, Czech Republic \\ ${ }^{3}$ Universidade Estadual Paulista, Rio Claro, SP, Brazil \\ Received 2014 February 18; accepted 2014 April 28; published 2014 June 23
}

\begin{abstract}
The Nice model of the dynamical instability and migration of the giant planets can explain many properties of the present solar system, and can be used to constrain its early architecture. In the jumping-Jupiter version of the Nice model, required from the terrestrial planet constraint and dynamical structure of the asteroid belt, Jupiter has encounters with an ice giant. Here, we study the survival of the Galilean satellites in the jumping-Jupiter model. This is an important concern because the ice-giant encounters, if deep enough, could dynamically perturb the orbits of the Galilean satellites and lead to implausible results. We performed numerical integrations where we tracked the effect of planetary encounters on the Galilean moons. We considered three instability cases from Nesvorný \& Morbidelli that differed in the number and distribution of encounters. We found that in one case, where the number of close encounters was relatively small, the Galilean satellite orbits were not significantly affected. In the other two, the orbital eccentricities of all moons were excited by encounters, Callisto's semimajor axis changed, and, in a large fraction of trials, the Laplace resonance of the inner three moons was disrupted. The subsequent evolution by tides damps eccentricities and can recapture the moons in the Laplace resonance. A more important constraint is represented by the orbital inclinations of the moons, which can be excited during the encounters and not appreciably damped by tides. We find that one instability case taken from Nesvorný \& Morbidelli clearly does not meet this constraint. This shows how the regular satellites of Jupiter can be used to set limits on the properties of encounters in the jumping-Jupiter model, and help us to better understand how the early solar system evolved.
\end{abstract}

Key words: planets and satellites: dynamical evolution and stability - planets and satellites: general - planets and satellites: individual (Jupiter, Galilean satellites)

Online-only material: color figures

\section{INTRODUCTION}

It is currently well accepted that the outer planets radially migrated in the past (Fernandez \& Ip 1996). In Hahn \& Malhotra (1999) and Tsiganis et al. (2005), two different migration histories were proposed. In the former, the planets were suggested to migrate in a smooth manner from their original orbits to those that they occupy today. The latter, instead, invoked a highly chaotic stage, where the outer planets underwent close encounters among themselves. Specifically, in the simulations of Tsiganis et al. (2005), the outer planets were initially located between 5 and $18 \mathrm{AU}$, and a massive outer planetesimal disk was placed beyond $20 \mathrm{AU}$. The instability was triggered in these simulations when Jupiter and Saturn migrated (by scattering planetesimals) over their mutual 2:1 mean motion resonance (MMR). During the instability, the orbits of Uranus and Neptune became Saturn-crossing, Uranus and Neptune were scattered out by Saturn, and these planets subsequently migrated to their current locations by gravitationally interacting with the outer disk. This model, also known as Nice model, appears to well explain many properties of the present solar system, such as the final orbital elements of the giant planets (Tsiganis et al. 2005), origin of the Late Heavy Bombardment (Gomes et al. 2005), capture of Jupiter's Trojans and the irregular satellites at Saturn, Uranus, and Neptune (Morbidelli et al. 2005; Nesvorný et al. 2007), origin of the dynamical structure of the Kuiper belt, and the implantation of primitive

\footnotetext{
4 Presently at Instituto Nacional de Pesquisas Espaciais, São José dos Campos, SP, Brazil.

5 Visiting Southwest Research Institute, Boulder, CO, USA.
}

trans-Neptunian objects into the outer asteroid belt (Levison et al. 2008, 2009).

However, as originally envisioned, the Nice model is unlikely to be correct in details. This is, for example, because the initial configuration of planets used in Tsiganis et al. (2005) is difficult to reconcile with the previous stage when planets formed and migrated in the protoplanetary gas disk. Instead, according to Morbidelli et al. (2007), Jupiter and Saturn should have emerged from the dispersing gas nebula with orbits in the 3:2 MMR (or possibly the 2:1 MMR), while Uranus and Neptune should have evolved onto nearby resonant orbits as well. The instability trigger proposed by Morbidelli et al. (2007) was the crossing of the 5:3 MMR between Jupiter and Saturn (later the crossing of the 2:1 MMR also occurs, but much faster than in Tsiganis et al. 2005). Another possible instability trigger was proposed by Levison et al. (2011), were the authors assumed a selfgravitating planetesimal disk. In that work, Levison et al. (2011) considered the delay between the dispersion of the proto-solar nebula and the instability. The planets were initially assumed to be locked in a multi-resonant state (Morbidelli et al. 2007), and the inner edge of the planetesimal disk was placed several AUs beyond the orbit of the outermost planet. Levison et al. (2011) modeled the disk's viscous stirring, induced by the presence of Pluto-sized objects embedded in the outer disk. They showed that viscous stirring leads to an irreversible exchange of energy between planets and the planetesimal disk. This exchange of energy induces the inward migration of the inner ice giant. As this planet is locked in resonance with Saturn, due to the adiabatic invariance, its eccentricity increases. During this process, the system crosses many weak secular resonances. Those resonances can disrupt the mean motion resonances and 
make the planetary system unstable long after the dispersal of the protoplanetary nebula.

Additional modifications of the original Nice model were motivated by the evolution of secular modes during planetary migration, mainly $g_{5}, g_{6}$, and $s_{6}$, and their effects on the terrestrial planets and asteroid belt. Brasser et al. (2009) found that it would be problematic if $g_{5}$ slowly swiped over the $g_{1}$ or $g_{2}$ modes of the terrestrial planets, because the $g_{1}=g_{5}$ and $g_{2}=g_{5}$ resonances would produce excessive excitation and instabilities in the terrestrial planet system (see also Agnor \& Lin 2012). Moreover, Morbidelli et al. (2010) and Minton \& Malhotra (2011) showed that the behavior of the $g_{6}$ and $s_{6}$ modes was crucially important for the asteroid belt, where, again, slow evolution of the $g_{6}$ and $s_{6}$ modes would violate constraints from the orbital distribution of asteroids.

To avoid these problems, it has been suggested that Jupiter's orbit should have discontinuously changed by encounters with an ice giant. The $g_{5}, g_{6}$, and $s_{6}$ frequencies are mainly a function of the orbital separation between Jupiter and Saturn. Thus, as Jupiter's semimajor axis moves in during encounters (and Saturn's semimajor axis moves out), these frequencies decrease in discrete steps. This kind of evolution is desirable, because the secular resonances with the terrestrial planets can be stepped over, and not activated. This instability model is known as the jumping-Jupiter model. Taken together, the initial conditions leading to the instability and the dynamical evolution of the planets were likely different from those originally envisioned in the Nice model.

Nesvorný (2011) and Nesvorný \& Morbidelli (2012) studied the possibility that more than four giant planets formed in the outer solar system. They showed that including a planet with mass comparable to that of Uranus or Neptune on an orbit between the original orbits of Saturn and Uranus can significantly increase the success rate of instability simulations. This is because, more often than not, at least one ice giant is ejected from the solar system during the instability. The fiveplanet cases considered in Nesvorný (2011) and Nesvorný \& Morbidelli (2012) showed just the right kind of the jumpingJupiter evolution discussed above, and also often satisfied various other constraints. The six-planet case worked as well but did not offer significant advantages over the five-planet case.

To understand the early evolution of the solar system it is important to not only consider the evolution of giant planets, but also to determine the effects of the planetary evolution on the populations of small bodies. Many recent works studied the effects of planetary migration on Jupiter and Neptune Trojans, asteroids and Kuiper belt objects, etc. (Morbidelli et al. 2005; Levison et al. 2008, 2009; Nesvorný \& Vokrouhlický 2009). Here, we consider the planetary satellites.

The satellites of the giant planets can be divided into several categories. The regular moons have orbits close to their host planet, and small orbital eccentricities and orbital inclinations with respect to the planet's equator. The irregular satellites, on the other hand, have distant orbits, and high eccentricities and high inclinations (as measured with respect to planet's orbital plane). It is believed that the irregular satellites were captured from heliocentric orbits. Nesvorný et al. (2007), for example, suggested that the irregular satellites were captured during encounters between planets in the Nice model when background planetesimals were deflected onto bound orbits. They showed that this type of capture has the right efficiency (up to a factor of few) to explain observations and leads to a roughly correct distribution of orbits of captured satellites at
Saturn, Uranus, and Neptune. However, because Jupiter does not generally participate in planetary encounters in the original Nice model (Nesvorný et al. 2007), where the encounter statistics were based on the original Nice model simulations, we were unable to address with their model the origin of the irregular satellites at Jupiter. The problem of the irregular satellite capture at Jupiter was recently reconsidered by us (Nesvorný et al. 2014a) in the context of the jumping-Jupiter model, where Jupiter participates in encounters.

The regular satellites represent a different concern. They presumably formed near their present orbits well before the instability, and were exposed to the effects of planetary encounters during the instability. Therefore, it is interesting to evaluate the degree of orbital excitation to determine whether the currently favored instability models are consistent with the systems of regular satellites we see at the outer planets today.

Our work described here builds on the previous efforts that considered the effect of planetary encounters on the regular satellites. Deienno et al. (2011) studied the history of planetary encounters in the instability model with four planets and found that the regular satellites at Uranus became destabilized in about $40 \%$ of the considered cases, if Uranus had close encounters with Saturn. Also, if these encounters occurred, any satellites beyond Oberon's orbit would have most certainly become unbound (Deienno et al. 2011).

The encounters between Uranus and Saturn do not typically occur in the instability model with five planets (Nesvorný \& Morbidelli 2012). The survival of Uranus's satellites is therefore less of a problem in the five-planet model (Nesvorný et al. 2014b). Instead, in this model, Saturn has encounters with the ejected ice giant. Interestingly, these planetary encounters can lead to the orbital excitation of Saturn's moons Titan and Iapetus, and may potentially explain, as shown in Nesvorný et al. (2014b), the anomalous inclination of Iapetus with respect to the Laplace surface (Tremaine et al. 2009).

Our main goal in this paper is to determine the orbital perturbations of the Galilean moons at Jupiter in the five-planet jumping-Jupiter models taken from Nesvorný \& Morbidelli (2012). This is an important issue because the third ice giant included in these simulations has many dozens of close encounters with Jupiter, and is ejected as a results of these encounters to interstellar space. The other planets that remain in the solar system (Saturn, Uranus, and Neptune) suffer fewer encounters with the ice giant; the perturbations of their regular satellites should therefore be less of an issue (see Nesvorný et al. 2014b). We also simulate the tidal evolution of the Galilean moons after the instability to show that their orbital eccentricities, if excited by the encounters, could have decreased by tides during the subsequent evolution.

The structure of this paper is as follows. Section 2 explains our method for tracking the planetary encounters and orbits of the regular satellites. In Section 3, we discuss the number and distribution of encounters in different instability cases and the orbital excitation of the Galilean moons during encounters. We also discuss in this section the tidal effects on the Galilean satellites and the behavior of the Laplace resonance. In Section 4, we summarize the main conclusions of this paper.

\section{METHODOLOGY}

We used three different cases of planetary instability taken from the simulations of Nesvorný \& Morbidelli (2012). We refer to these cases as Case 1, Case 2, and Case 3. They are the 
same cases as the ones used in the previous studies of capture of Jupiter's Trojans and irregular satellites (Nesvorný et al. 2013, 2014a). By using the same cases as in the previous works, we aim to subject the selected instability scenarios to a number of tests. Our hope is to advance toward a fully self-consistent model of the early solar system instability.

The three selected cases feature three different histories of encounters of Jupiter with ice giants, resulting in different dynamical perturbations of the Galilean satellites. We have considered planetary encounters whenever the distance between the planets was less than the sum of their Hill radii $(d<$ $R_{\mathrm{Hill}}^{\mathrm{Jup}}+R_{\mathrm{Hill}}^{\mathrm{Ice}}$ ). The original simulations in Nesvorný \& Morbidelli (2012) were repeated and we recorded all encounters of Jupiter that satisfied the above criteria. See Section 3.1 for a discussion of encounters in each case.

The configuration of the Galilean satellites at the onset of the planetary instability is unknown. To set up our simulations, we opted to use the current orbits of Io, Europa, and Ganymede from JPL Horizons ${ }^{6}$ at ten slightly different epochs. This choice implies that our initial satellite configurations have the inner three moons in the Laplace resonance, and we can test, among other things, how the Laplace resonance is affected by planetary encounters. Callisto was placed on an orbit with Callisto's present semimajor axis, and zero eccentricity and zero inclination to Jupiter's equator. To increase the statistics, we considered a hundred different positions of Callisto along the orbit for each one of the ten epochs. The mean longitude $\ell$ was set such that $\ell=k \Delta \ell$, where integer $k=0,1, \ldots, 99$ and $\Delta \ell=3$.6. Thus, in total, we have 1000 different configurations of the Galilean satellites for each case. In addition, we also considered an additional set of 1000 initial configurations, where Callisto was placed in the outer 2:1 MMR with Ganymede. This was done to test whether it is possible that all four Galilean satellites started in a chain of mean motion resonances, and Callisto was kicked out of the resonance with Ganymede by planetary encounters.

We proceed as follows. (1) Starting from the planetary positions and velocities recorded at the first encounter, the system containing the Sun and planets is integrated backward until the separation of Jupiter and the ice giant reaches 2 AU (let $\Delta t$ be the time for this to happen). (2) The Galilean satellites are placed at Jupiter on orbits described above. (3) We then integrate the orbits of planets and the Galilean satellites forward for time $2 \Delta t+P_{J}$, where $P_{J}$ is the orbital period of Jupiter (we also tested timespans of 10 and 100 times $P_{J}$ to verify that the results do not depend on our assumptions). The effects of Jupiter's oblateness $\left(J_{2}\right)$ and obliquity $\left(\varepsilon \simeq 3^{\circ}\right)$ are included in these integrations (precession of Jupiter's spin vector is ignored). (4) The orbits of the Galilean satellites obtained at this point are used as the initial orbits for the next encounter and the procedure is iterated over all encounters. (5) Finally, the satellite orbits after the last encounter are integrated for an additional $1000 \mathrm{yr}$. We use this simulation to compute the mean orbital elements of the Galilean satellites. As the encounters happen in a narrow window of time, tidal damping in between encounters is negligible. All the integrations described above were conducted using the Bulirsch-Stoer integrator from the Mercury code (Chambers 1999).

Before the computed mean elements can be compared with the real mean elements of the Galilean satellites in the present solar system, we must account for the orbital evolution of the

\footnotetext{
6 telnet://horizons.jpl.nasa.gov:6775 (terminal access).
}

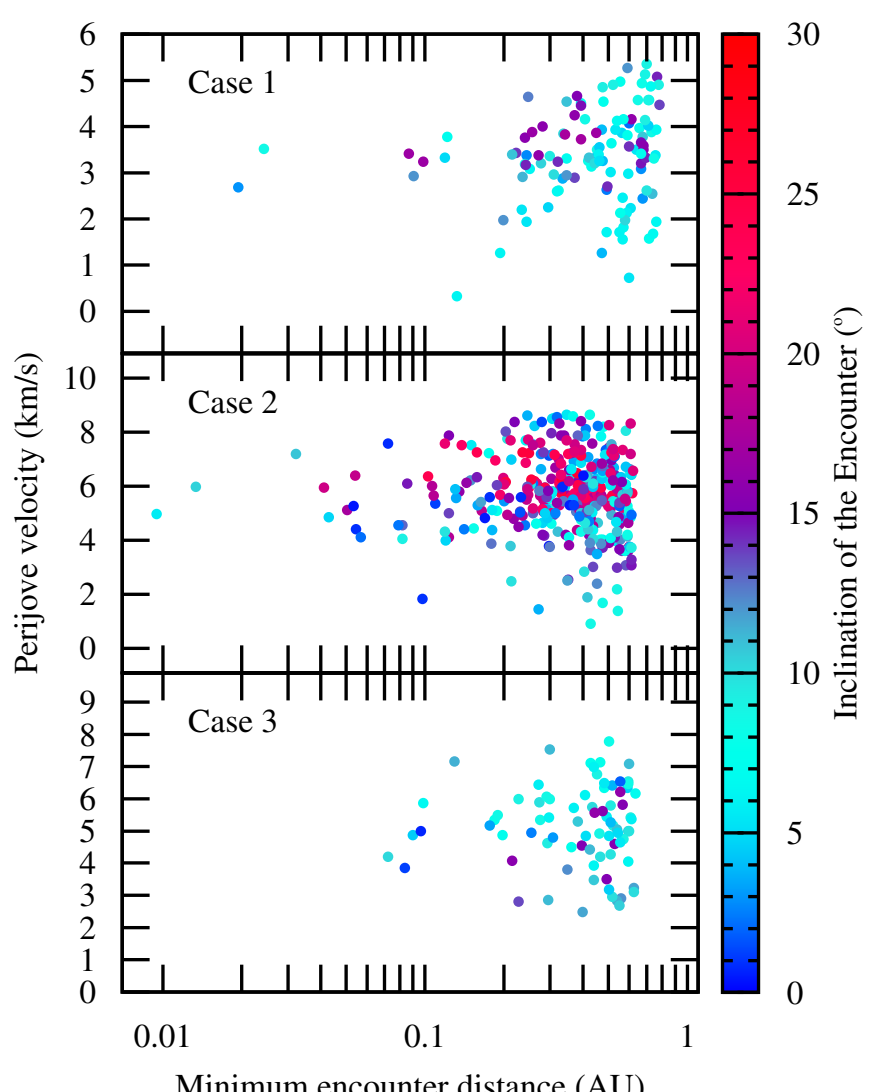

Figure 1. Perijove velocity, inclination (with respect to Jupiter's orbit), and minimum distance of each recorded encounter in Cases 1, 2, and 3.

(A color version of this figure is available in the online journal.)

Galilean satellites from the time of instability to the present. Considering that the planetary instability most likely occurred about 4 Gyr ago, the tidal effects can be particularly important. To study tidal evolution, we modified the symplectic integrator known as swift rmvs3 (Levison \& Duncan 1994) to include the tidal acceleration terms from Mignard (1979). The tidal dissipation in this model depends the Love number $k_{2}$ and dissipation parameter $Q$. The values of these parameters for Jupiter and the Galilean satellites are poorly known. Lainey et al. (2009) found, by fitting a tidal model to the astrometric observations of the Galilean satellites, that $k_{2} / Q=1.102 \times 10^{-5}$ for Jupiter and $k_{2} / Q=0.015$ for Io. We adopt these values here, and for simplicity keep them constant throughout the evolution. The results of our tidal simulations are presented in Section 3.4.

\section{RESULTS}

\subsection{Properties of Encounters}

Figure 1 shows the minimum distance between planets, perijove velocity of the ice giant, and trajectory inclination for all planetary encounters of Jupiter. The number and geometry of encounters differ from case to case. The number of encounters is 117, 386, and 80 in Cases 1, 2, and 3, respectively. The number of encounters reaching minimum distance $d_{\min }<0.05$ AU is 2, 9, and 0 in these cases. As we will see below, these deep encounters matter the most for the excitation of the Galilean satellites. Most encounters have perijove velocities roughly within the range from 3 to $6 \mathrm{~km} \mathrm{~s}^{-1}$ and inclinations smaller than $10^{\circ}$. Only Case 2 shows inclinations up to $30^{\circ}$. 


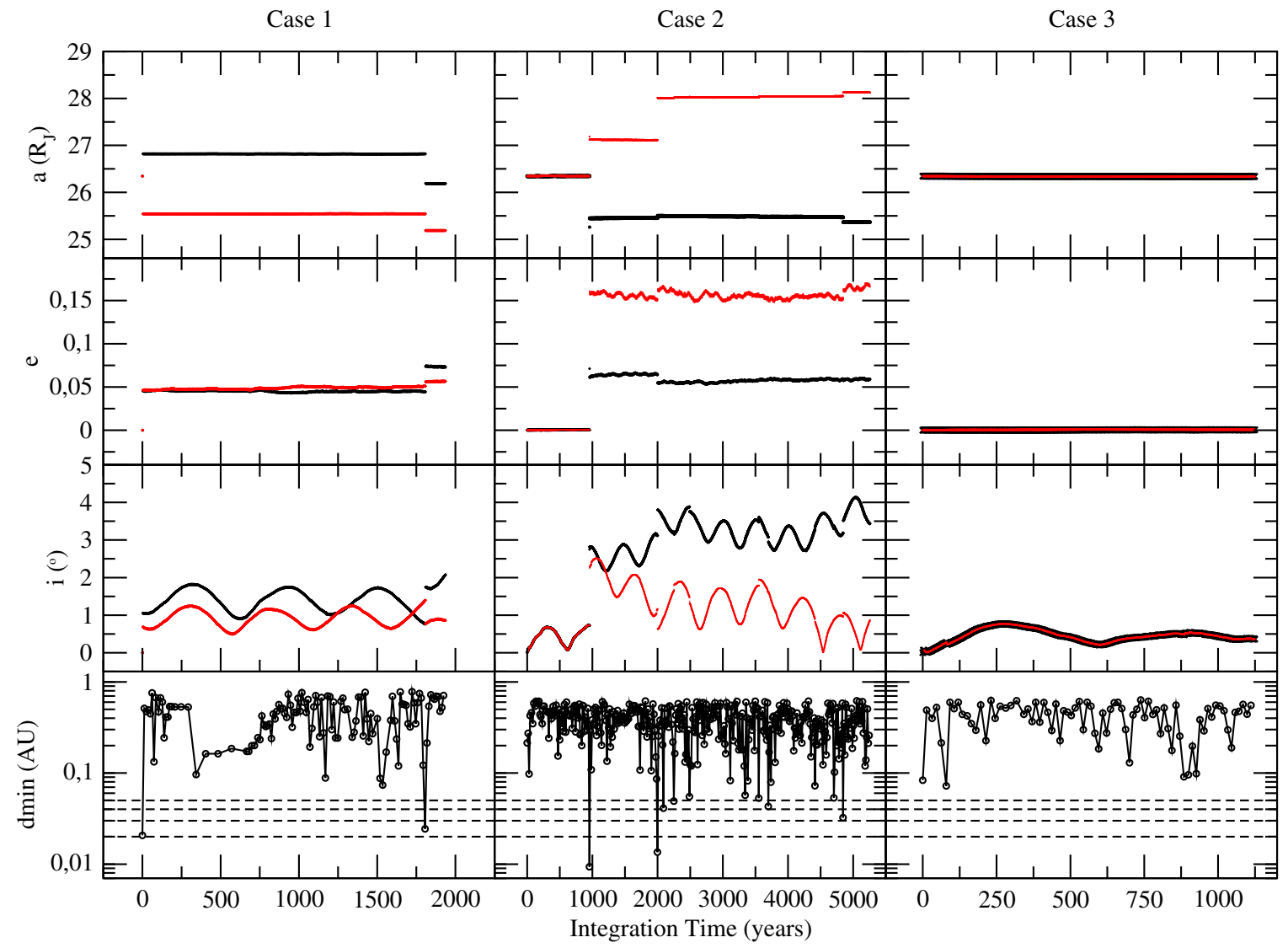

Figure 2. From top to bottom: evolution of the semimajor axis, eccentricity, orbital inclination (relative to the equatorial plane of Jupiter), and the minimum distance of encounters as a function of the integration time (integration time $=n \times\left[2 \Delta t+P_{J}\right], n=1 \ldots N_{\text {enc }}$ ), for two different phases of Callisto on its orbit (red and black). From left to right: Cases 1,2, and 3. The dashed lines in the bottom panels, from top to bottom, represent four values of minimum encounter distance from $0.05 \mathrm{AU}$ to $0.02 \mathrm{AU}$.

(A color version of this figure is available in the online journal.)

Figure 2 shows an example of orbital perturbations of Callisto's orbit obtained in our simulations. In each case, the results are shown for two different initial phases of Callisto. The figure illustrates that the effect of encounters can lead to different outcomes. In some encounters, the satellite eccentricity, inclination, and semimajor axis increase, while in other encounters the values of orbital elements decrease.

The degree of perturbation during an encounter apparently depends on the minimal distance $d_{\min }$. In general, the encounters with $d_{\min }>0.05 \mathrm{AU}$ do not cause significant variations (see Case 3 in Figure 2). The encounters with $0.03<d_{\min }<$ $0.05 \mathrm{AU}$ can lead to relatively small changes in the orbital inclinations, but otherwise do not affect orbital elements much. The encounters with $0.02<d_{\min }<0.03 \mathrm{AU}$, on the other hand, are already deep enough to significantly perturb the orbits of satellites, mainly that of Callisto (for reference, Callisto's semimajor axis is $\simeq 0.012 \mathrm{AU}$ ). Only very few of these encounters can happen if major excitation of Callisto's orbit is to be avoided. Finally, the encounters with $d_{\min }<0.02 \mathrm{AU}$ are the most destructive in that they lead to a major excitation of the satellite orbits, and, in some cases, can eject Callisto from the system.

\subsection{Excitation of Satellite Orbits}

\subsubsection{Case 1}

Case 1 is an intermediate case with 117 total encounters, two of which have $0.02<d_{\min }<0.03$ AU (Figures 1 and 2).

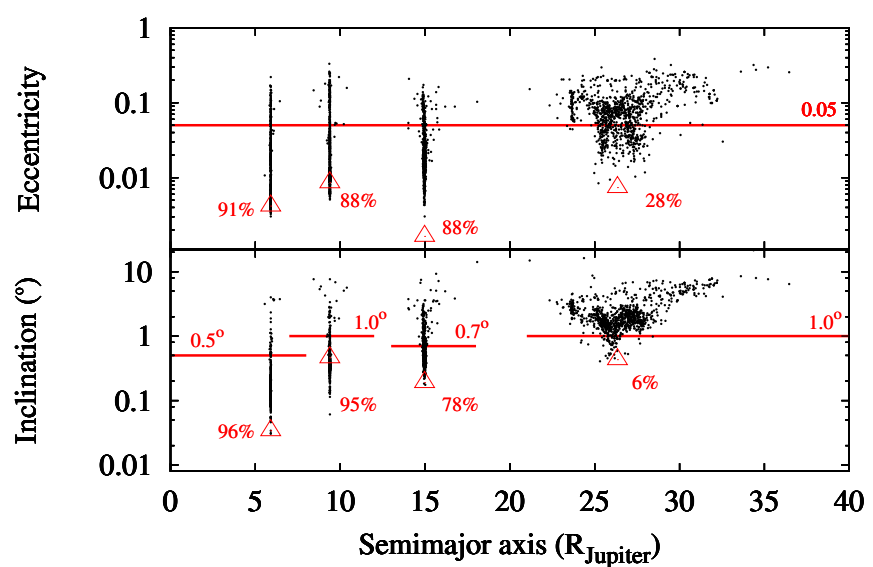

Figure 3. Case 1. Top: final averaged eccentricity as a function of the final averaged semimajor axis. Bottom: the final averaged orbital inclination with respect to Jupiter's equator. The simulation results are shown by black dots. The triangles show the current averaged values of the orbital elements of the Galilean moons (from left to right: Io, Europa, Ganymede, and Callisto). The labels denote the percentage of simulated trials that ended up below the reference lines (discussed in the main text).

(A color version of this figure is available in the online journal.)

Figure 3 summarizes the results of our simulations in Case 1. For reference, as a simple criterion of the plausibility of the results, we plot in this figure the lines $e_{\text {ref }}=0.05$ for eccentricities, and $i_{\text {ref }}$ for inclinations, where $i_{\text {ref }}$ was chosen to be 0.5 greater than 


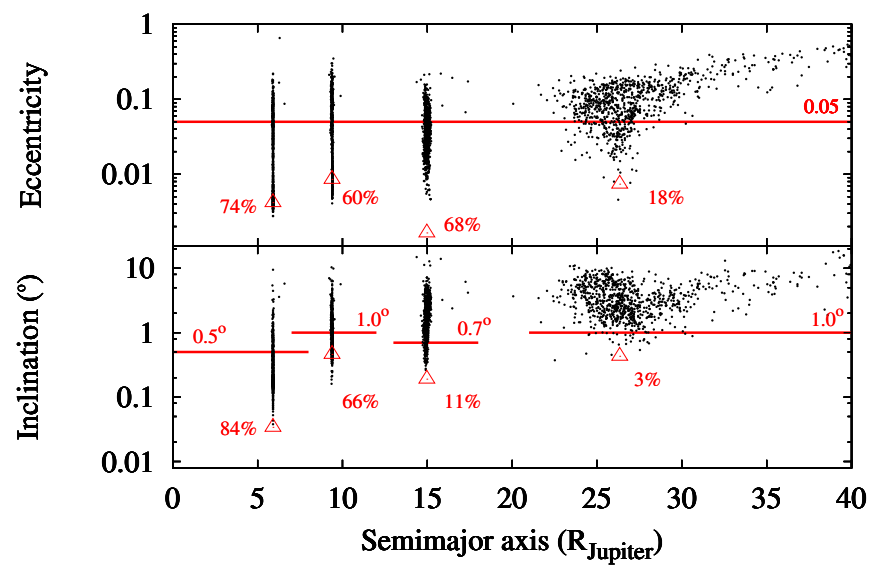

Figure 4. Same as Figure 3 but for Case 2.

(A color version of this figure is available in the online journal.)

the current mean inclination. We calculate the fraction of final orbits that end up having $e<e_{\text {ref }}$ and $i<i_{\text {ref }}$. These fractions are shown in Figure 3.

The orbits of the inner three satellites are only modestly excited by the encounters. The fraction of trials in which the eccentricities and inclinations end up below the reference values is large ( $>75 \%$ and $\gtrsim 90 \%$, respectively). These results are plausible. A more stringent constraint is represented, as expected, by Callisto's orbit. We find that about one-third of the trials end up with Callisto's eccentricity $e<e_{\text {ref }}$. This is, however, still a relatively large fraction. Moreover, as we will see in Section 3.4, Callisto's and other moon's eccentricities can be damped by tides after the stage of planetary encounters. The eccentricity excitation cannot therefore be used, in general, to rule out specific encounter histories (except if orbits become unbound). The results for Callisto's inclination are more interesting, because only a very small fraction of trials end up with $i<i_{\text {ref }}$ (Figure 3), and the inclination remains nearly unchanged during the subsequent tidal evolution (Section 3.4).

As for the effect of encounters on the semimajor axis, the inner three satellites end up very near their original semimajor axis values. Even if the changes are small they can have a major consequence for the Laplace resonance in that, at least in some cases, the libration amplitude of the Laplace resonance can significantly increase or the moons can end up on non-resonant orbits. We will discuss this issue in Section 3.5.

The semimajor axis of Callisto can change substantially (by up to $\simeq 5 R_{\text {Jupiter }}$, where Jupiter's equatorial radius $R_{\text {Jupiter }} \simeq$ $71500 \mathrm{~km}$ ). This raises a question of whether Callisto could have originally shared the Laplace resonance with the inner moons (the 2:1 MMR with Ganymede is located at $a \simeq 23.76$ $R_{\text {Jupiter }}$ ), and was scattered to its current orbit ( $a \simeq 26.33 R_{\text {Jupiter }}$ ) by encounters. We address this issue in Section 3.3.

\subsubsection{Case 2}

Figure 4 shows orbital perturbations of the Galilean satellites in Case 2. In this case, we have nine encounters with $d_{\min }<$ $0.05 \mathrm{AU}$ and two encounters with $d_{\min }<0.02 \mathrm{AU}$. These close encounters produce very large perturbations of orbits of the Galilean satellites. For example, in only $11 \%$ of trials Ganymede's orbital inclination ends up below our reference value $(i<0.7)$, and in only $3 \%$ of trials this criterion holds for Callisto's inclination. The changes to Io's and Europa's orbits

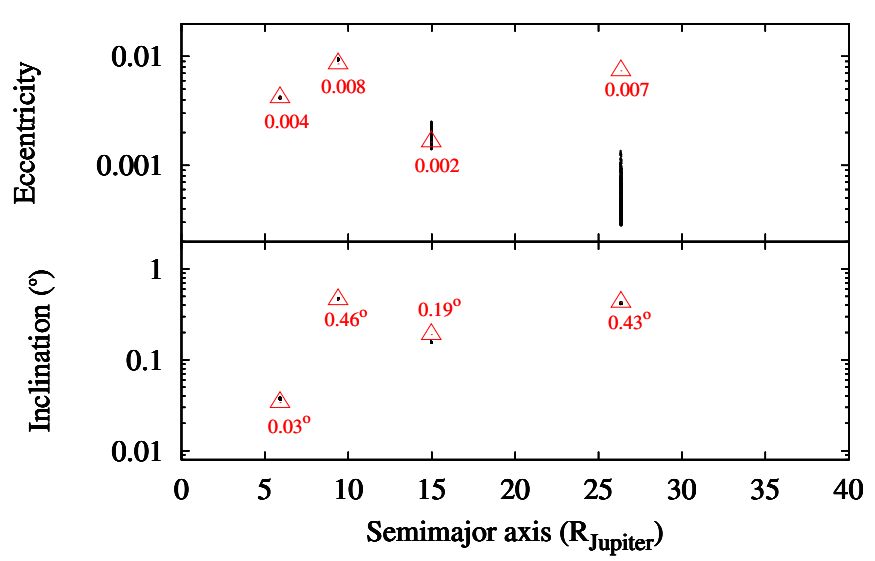

Figure 5. Same as Figure 3 but for Case 3. The numbers denote the average values of the eccentricity and inclination (with respect to the planet equator) of the Galilean moons.

(A color version of this figure is available in the online journal.)

are also substantial. Given these results, we believe that this case can be ruled out.

\subsubsection{Case 3}

Figure 5 summarizes the outcomes of our simulations in Case 3. In this case, there were no planetary encounters with $d_{\text {min }}<0.05$ AU and orbits of the Galilean moons did not change much. Therefore, Case 3 is clearly plausible and would imply that the architecture of the Galilean system has remained nearly unchanged during the instability.

An interesting observation, unrelated to planetary encounters, concerns Callisto's inclination. Recall that we started with Callisto's orbit with zero inclination with respect to Jupiter's equatorial plane. It may then seem surprising, as seen in Figure 5, that mean orbital inclination with respect to the equatorial plane ends up exactly matching the present value of Callisto $(i=0.43)$. This result arises because the Laplace surface at the location of Callisto's orbit is inclined by $\simeq 0.43$ to Jupiter's equator (Ward \& Canup 2006; Tremaine et al. 2009). Thus, an orbit starting on the equatorial plane will have oscillations, with an amplitude reaching almost $1^{\circ}$ in the equatorial reference plane, and an average inclination value equal to the tilt between equatorial and Laplace planes. On the other hand, the oscillation of Callisto's orbital inclination with respect to the Laplace plane is negligible and follows the evolution of the Laplace surface during the evolution of Jupiter's semimajor axis. This observation may have interesting implications for the origin of Callisto's orbital inclination, because it shows that Callisto's orbit should have initially coincided with Jupiter's equator, as expected, for example, in the presence of a heavy circumplanetary disk, or if Jupiter's obliquity was zero. This would require that the circumplanetary disk was dispersed, or the Jupiter's obliquity was tilted, on a very short timescale $\left(\sim 10^{2} \mathrm{yr}\right)$.

\subsection{Tests with Callisto in a Resonance}

With Callisto's semimajor axis considerably changing in Cases 1 and 2, we decided to test a possibility that Callisto's orbit was initially in the 2:1 MMR with Ganymede (potentially driven there when satellites radially migrated by interacting with the circumplanetary gas nebula, or when Ganymede was pushed out by tides; Yoder \& Peale 1981; Canup \& Ward 2002, 2006, 2009), 


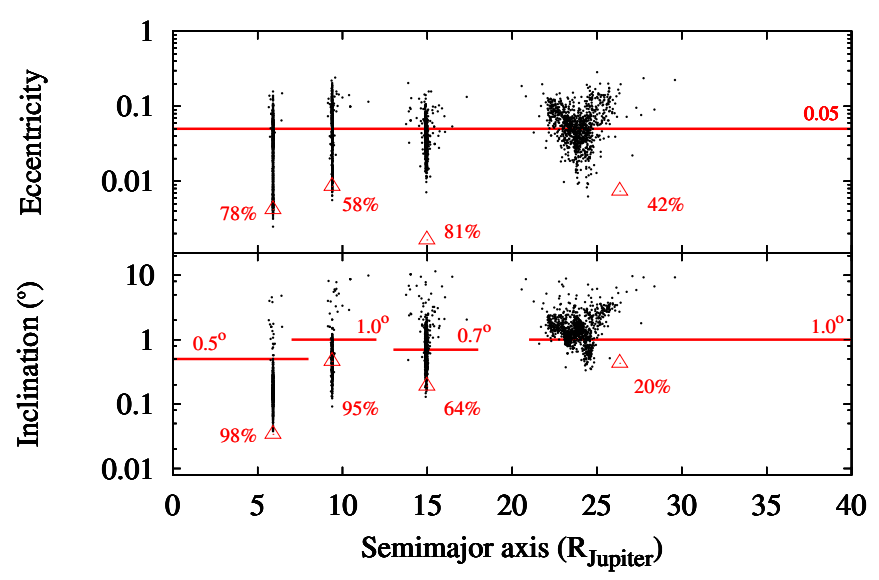

Figure 6. Same as Figure 3 but starting with all four Galilean satellites in a chain of the 2:1 MMRs (i.e., Callisto in the 2:1 MMR with Ganymede).

(A color version of this figure is available in the online journal.)

and Callisto was kicked out of the resonance during planetary encounters. These tests were done for Case 1, because this case leads to a large variation of Callisto's semimajor axis, as needed to move Callisto's orbit from the resonance to its current location (without any large eccentricity and/or inclination excitation seen in Case 2).

The initial orbits of the Galilean satellites were set by the method described in Section 2, except that Callisto was placed in the 2:1 MMR orbit with Ganymede. We find that these new simulations produce results very similar to those obtained with the original initial conditions (compare Figure 6 with Figure 3). The fraction of trials in which Callisto's eccentricity and inclination ended up below the reference values are now higher than before ( $42 \%$ and $20 \%$, respectively). This is related to a strong gradient of the excitation pattern with the radial distance from Jupiter and the fact that Callisto started with a slightly smaller semimajor axis value in these new simulations.

Figure 6 shows that, while it is plausible to change Callisto's semimajor axis by the required amount, the eccentricity and inclination are typically excited quite a bit for encounter distances $0.02<d_{\min }<0.03 \mathrm{AU}$, such that only a negligible fraction of trials end up with $a \simeq 27 R_{\text {Jupiter, }}$ and $e<e_{\text {ref }}$

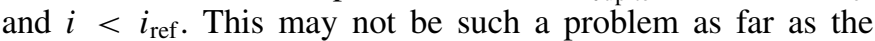
eccentricity is concerned, because Callisto's eccentricity could have been damped by tides during the subsequent evolution (see Section 3.4).

Its orbital inclination, however, is almost unaffected by tides, and thus presents a more rigid constraint. In particular, we do not see how the excited orbits with $a \simeq 27 R_{\text {Jupiter }}$ and $i>3^{\circ}$ (Figure 6) could reach $i<1^{\circ}$ to become a plausible proxy for the current Callisto orbit. We conclude that it is unlikely Callisto could have reached its current orbit by starting in the 2:1 MMR with Ganymede, being kicked out of the resonance by planetary encounters, and subsequently evolving by tides. Additional processes responsible for damping Callisto's inclination would have to be identified for this possibility to become viable.

\subsection{Tidal Evolution}

In the previous sections, we determined orbital perturbations of the Galilean satellites during close encounters in the jumpingJupiter model. These perturbations happened most likely some 4 Gyr ago. A question therefore arises of whether (and how) the satellite orbits could have changed during the $\sim 4$ Gyr period between the instability and the present time. Here, we consider the orbital changes produced by the tidal interaction of moons with Jupiter.

To achieve this goal, we performed a number of numerical integrations with a symplectic $N$-body code known as swift rmvs3 (Levison \& Duncan 1994) that we modified to include the tidal acceleration terms from Mignard (1979; see also Lainey et al. 2009, Equations (1) and (2) in their supplement). These simulations were performed in a reference system centered on Jupiter. We considered both the planetary and satellites tides. We found that the tidal dissipation in the system is overwhelmingly due to the interaction between Io and Jupiter. The direct tidal effects of other satellites can be neglected, but important indirect effects arise on these satellites because they are coupled with the Jupiter-Io pair. Therefore, Callisto's orbital eccentricity can be damped as a consequence of the strong dissipation in the pair Jupiter-Io, similar to what happens in the case of extrasolar planets, when damping is applied to the innermost planet in a planetary system (Lovis et al. 2011; Van Laerhoven \& Greenberg 2013).

The satellite rotation was assumed to be synchronous. To implement the synchronous rotation in the code, we adopted the following approximation (V. Lainey 2013, private communication). First, we considered only the radial component of the tidal acceleration that results from changing planetocentric distance (and the related dissipation). The radial component does not depend on satellite's rotation rate, and is therefore independent of the detailed assumptions about synchronicity. The longitudinal tidal acceleration is then effectively included by multiplying the strength of the radial tide by $7 / 3$. This is because in the limit of small eccentricities, which is applicable here, the orbital energy dissipated in satellite's librations is $4 / 3$ of that dissipated in radial flexing (e.g., Murray \& Dermott, 1999, Chapter 4).

The strength of tidal dissipation is parameterized by the time delay due to tidal response of the deformed body or equivalently by $k_{2} / Q$, where $Q$ is the dissipation function and $k_{2}$ is the Love number. We assume $k_{2} / Q=1.102 \times 10^{-5}$ for Jupiter and $k_{2} / Q=0.015$ for Io (Lainey et al. 2009), and evolve the system for 4 Gyr. Possible variations of $k_{2} / Q$ over the age of the solar system are not considered, because we are not interested in detailed modeling of the tidal evolution, but rather in the average magnitude of the tidal effect in 4 Gyr.

Figure 7 shows the eccentricity of the four Galilean satellites affected by tides in 4 Gyr. Most notably, as previously envisioned, we find that Callisto's eccentricity can be significantly damped through the coupling to the inner moons resonant system. In the specific case shown in Figure 7, Callisto's eccentricity decreases from 0.05 to 0.025 . This change should not be taken at its face value because the parameters of the tidal model are uncertain. The eccentricity drop can be a factor of several smaller, if the effective $k_{2} / Q$ of Io was lower than considered here, or a factor of several larger, if the effective $k_{2} / Q$ was higher. What this shows is that Callisto's eccentricity could have been significantly damped by tides over 4 Gyr. Conversely, Figure 8 shows that the orbital inclination of the satellites does not appreciably change during the tidal evolution.

These results have major implications for the interpretation of our scattering experiments discussed in the previous sections. First, they show that Callisto's eccentricity cannot be used as a rigid constraint on the instability models, because even if Callisto's eccentricity was significantly excited during planetary encounters, it could have decreased to the current value during the subsequent evolution. The orbital inclinations of the moons, on the other hand, represent a more useful constraint on the planetary encounters, and can be used to rule out specific 
Io
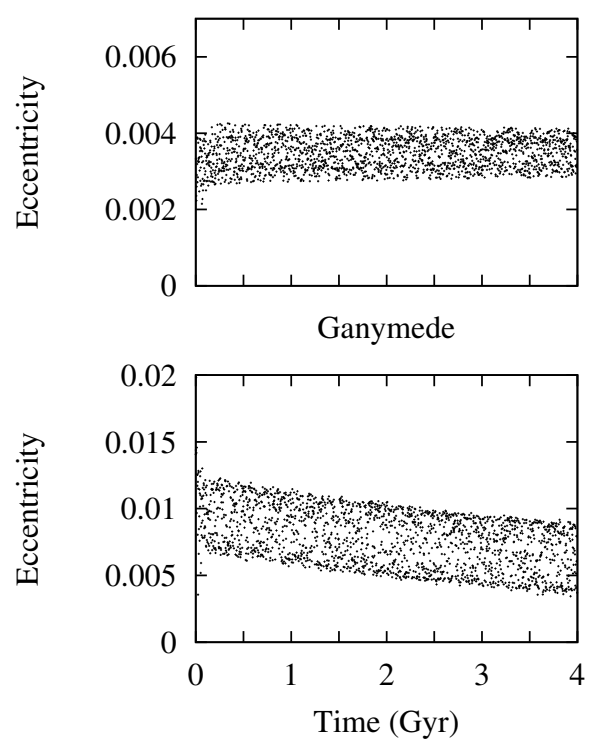

Europa

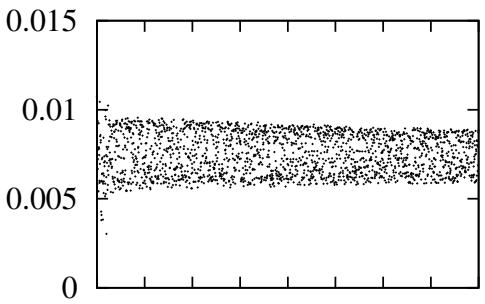

Callisto

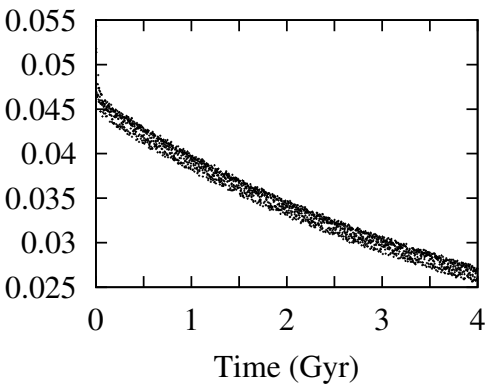

Figure 7. Evolution of the orbital eccentricity of the Galilean satellites during the tidal evolution.
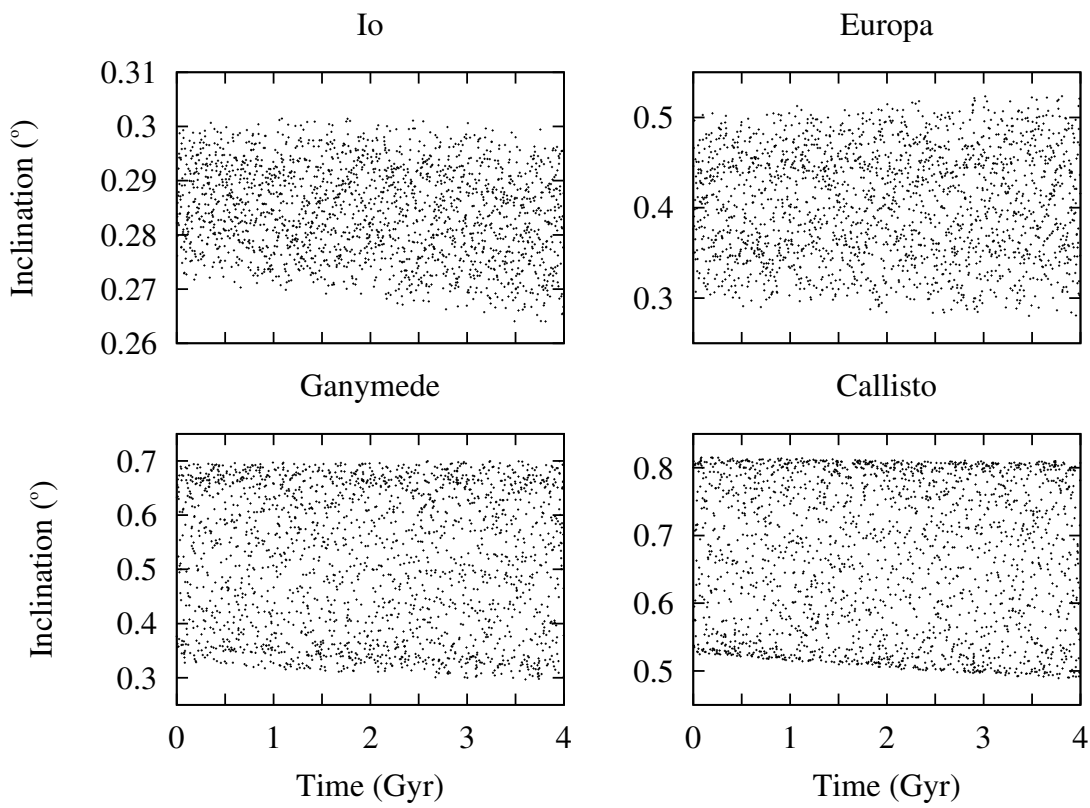

Figure 8. Evolution of the orbital inclination (with respect to Jupiter's equatorial plane) of the Galilean satellites during the tidal evolution.

instability models. For example, Case 2 discussed above is clearly implausible because Callisto's inclination remains reasonably low only in a few percent of trials.

As for the semimajor axes of the satellites, after several experiments with their different initial values we determined that the global result does not change much by changing semimajor axes. So, while the semimajor axes of the Galilean satellites can somewhat change in some cases, we believe that it should be enough to point out that tidal migration of Callisto was small and insufficient to resolve the problem in Figure 6.

\subsection{Laplace Resonance}

Another useful constraint on the instability models is represented by the fact that Io, Europa, and Ganymede are in the Laplace resonance. This is because, if the semimajor axes of these satellites change as a result of the planetary encounters, the orbits can end up escaping from the resonance. If this happens, the resonant angle $\phi_{L}=\lambda_{I}-3 \lambda_{E}+2 \lambda_{G}$, where $\lambda_{I}, \lambda_{E}$, and $\lambda_{G}$ are the mean longitudes of Io, Europa, and Ganymede, respectively, starts to circulate. By analyzing our simulations we found that this happened in $47 \%, 93 \%$, and $0 \%$ of the trials in Cases 1, 2, and 3, respectively.

These results show that Case 2 is problematic, as already pointed out above based on considerations related to the orbital inclination of Callisto. The Laplace resonance constraint, however, is not as solid as the inclination constraint, because tides could re-capture satellites in the Laplace resonance. For example, if Europa and Ganymede have roughly the same probability to be scattered inward and outward, we find that the outward tidal evolution of Io could re-capture Europa and Ganymede in the Laplace resonance in up to $25 \%$ of cases. This means, for example, that in up to roughly $65 \%$ of trials in Case 1, 


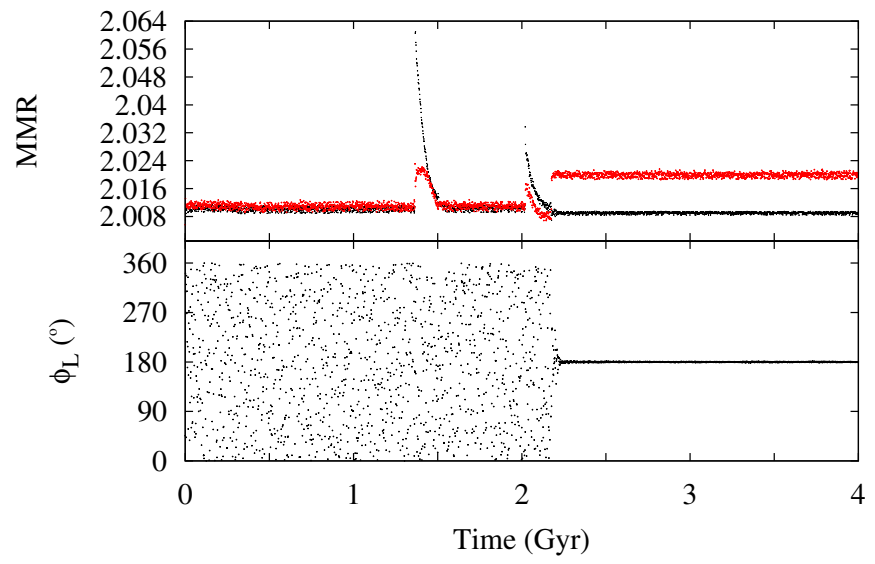

Figure 9. Top: evolution of the mean motion ratios of Io and Europa (black), and Europa and Ganymede (red). Bottom: evolution of the Laplace resonance angle $\phi_{L}=\lambda_{I}-3 \lambda_{E}+2 \lambda_{G}$. This figure illustrates that it is possible to break the Laplace resonance during planetary encounters, such that $\phi_{L}$ circulates at the beginning of our tidal simulations, and re-capture the inner three moons into the Laplace resonance when Io moves out by tides. In this specific case, the capture occurred at $\simeq 2.2$ Gyr when $\phi_{L}$ started librating around $180^{\circ}$.

(A color version of this figure is available in the online journal.)

the Laplace resonance could have been left undisturbed or was re-established during the subsequent tidal evolution. An illustration of a case where the moons were re-captured in the Laplace resonance is shown in Figure 9.

\section{CONCLUSIONS}

Here, we studied the orbital behavior of the Galilean satellites during the dynamical instability in the outer solar system. By carrying out numerical simulations, we determined the level of orbital perturbation in three instability cases taken from Nesvorný \& Morbidelli (2012). The orbital perturbations occur when Jupiter has a close encounter with an ice giant, and the gravity of the ice giant acts on the satellite orbits. We also studied the subsequent evolution of the surviving satellites due to the tidal effects.

We found that the orbits of the Galilean satellites can be profoundly affected by the planetary encounters between Jupiter and an ice giant, especially in the cases where the encounter distance $0.02 \mathrm{AU}<d_{\text {enc }}<0.03 \mathrm{AU}$. The extremely deep encounters with distance $d_{\text {enc }}<0.02$ AU can be clearly ruled out, because those would lead to strong orbital excitation, collisions of moons, and their removal. On the other hand, encounters with $0.03 \mathrm{AU}<d_{\mathrm{enc}}<0.05 \mathrm{AU}$ cause only small variations of the orbital elements, and those with $d_{\mathrm{enc}}>0.05$ AU leave the satellite system essentially undisturbed.

The subsequent tidal evolution of the moons, principally due to the tidal dissipation in the Jupiter-Io pair, is capable of damping Callisto's orbital eccentricity. Therefore, even if Callisto's eccentricity becomes excited by planetary encounters, it can decrease to values similar to Callisto's present eccentricity $(\simeq 0.03)$ later. Similarly, even if the Laplace resonance of the inner three moons can be broken during planetary instability, it can be reestablished later when Io moves tidally outward, and recaptures Europa and Ganymede in outer MMRs. Together, this means that the eccentricity and Laplace resonance do not represent stringent constraints that could be used to rule out specific instability models.

Conversely, the orbital inclinations of the Galilean moons, and mainly that of Callisto, provide an important constraint.
This is because the inclinations are nearly unaffected by tides. Therefore, if the inclinations would have been strongly excited during planetary encounters, such as in the Case 2 discussed earlier, they would survive to the presently. These cases could clearly be ruled out when compared to the currently low orbital inclinations of the Galilean satellites. Specifically, we find that any planetary encounters with $d_{\text {enc }}<0.02$ AU (Case 2) can clearly be ruled out, and that encounters with $0.02 \mathrm{AU}$ $<d_{\text {enc }}<0.03$ AU (Case 1) cannot be too many.

Encounters with $d_{\mathrm{enc}}>0.03$ AU can happen more often. This is interesting because these more distant encounters can be important for capturing the irregular satellites at Jupiter (Nesvorný et al. 2014a). We therefore find that the Galilean and irregular satellites represent different constraints on the planetary encounters: the former limit the distance and number of the few closest encounters, while the latter require that many distant encounters occur. These constraints are complementary and should ideally be used together when evaluating the plausibility of a specific instability model. For example, applying these constraints in Cases 1, 2, and 3 studied here and in Nesvorný et al. (2014a), we find that Case 3 is clearly plausible, Case 2 is clearly implausible, and Case 1 stands in the middle, where most parameters are acceptable except for Callisto's inclination, which ends up too high in most trials.

Still, orbital inclinations could have been damped by another mechanism such as, for example, dynamical friction exerted by a disk of debris (Deienno et al. 2012; R. Deienno \& T. Yokoyama, in preparation). In this case, Callisto could interact with a disk of debris and its orbital inclination be damped together with the other orbital elements. Such a disk could have formed if one considers that Jupiter could have had more satellites in the past than it has today (beyond Callisto's orbit), and those extra satellites were lost by collisions among themselves during the instability phase of the solar system (as proposed for Uranus' satellites by Deienno et al. 2011), or by a residual mass produced from collisional grinding of irregular satellites (Bottke et al. 2010). This could be an interesting alternative, because as shown by Ćuk \& Gladman (2005), dynamical friction is more effective in damping Triton's orbit than tides alone (working on inclination as well). However, such a hypothesis needs to be carefully analyzed to determine whether or not the mass and life time of the disk are plausibly large to affect Callisto's inclination. We leave this work for future investigations.

In a broader scope, while the Galilean satellite constraints discussed here can be used to rule out specific instability models, they cannot be used to rule out the jumping-Jupiter model in general. This is because many (but not all) previously developed jumping-Jupiter models should satisfy the Galilean satellite constraint as well. There is also the possibility that the initial giant planet system had more than five planets, where the additional planets were smaller in mass (Nesvorný \& Morbidelli 2012). In this case, satellite excitation would be presumably less severe than found in Cases 1 and 2 in this paper. This result should be seen in positive light, because the jumping-Jupiter model is required from the terrestrial planet constraint (Brasser et al. 2009; Morbidelli et al. 2007; Nesvorný \& Morbidelli 2012), the dynamical structure of the asteroid belt (Morbidelli et al. 2010), and for the capture of the irregular satellites at Jupiter (Nesvorný et al. 2014a).

This work was supported by FAPESP (grants 2012/237324 and 2010/11109-5). D.N. was supported by NASA's Outer Planet Research program. The work of D.V. was partly 
supported by the Czech Grant Agency (grant P209-13-013085). T.Y. was supported by CNPq. We thank an anonymous referee for useful comments on the submitted manuscript.

\section{REFERENCES}

Agnor, C. B., \& Lin, D. N. C. 2012, ApJ, 745, 143

Bottke, W. F., Nesvorný, D., Vokrouhlický, D., \& Morbidelli, A. 2010, AJ, 139,994

Brasser, R., Morbidelli, A., Gomes, R., Tsiganis, K., \& Levison, F. H. 2009, A\&A, 134, 1790

Canup, R. M., \& Ward, W. R. 2002, AJ, 124, 3404

Canup, R. M., \& Ward, W. R. 2006, Natur, 441, 834

Canup, R. M., \& Ward, W. R. 2009, in Europa, ed. R. T. Pappalardo et al. (Tucson: Univ. Arizona Press), 59

Chambers, J. E. 1999, MNRAS, 304, 793

Ćuk, M., \& Gladman, B. J. 2005, ApJL, 626, L113

Deienno, R., Yokoyama, T., Nogueira, E. C., Callegari, N., Jr., \& Santos, M. T. 2011, A\&A, 536, A57

Deienno, R., Yokoyama, T., \& Prado, A. F. B. A. 2012, AAS/Division for Planetary Sciences Meeting Abstracts, 44, 415.09

Fernandez, J. A., \& Ip, W. H. 1996, P\&SS, 44, 431

Gomes, R. S., Tsiganis, K., Morbidelli, A., \& Levison, H. F. 2005, Natur, 435,466

Hahn, J. M., \& Malhotra, R. 1999, AJ, 117, 3041

Lainey, V., Arlot, J., Karatekin, Ö., \& Hoolst, T. V. 2009, Natur, 459, 957

Levison, H. F., Bottke, W. F., Gounelle, M., et al. 2009, Natur, 460, 364
Levison, H. F., \& Duncan, M. J. 1994, Icar, 108, 18

Levison, H. F., Morbidelli, A., Tsiganis, K., Nesvorný, D., \& Gomes, R. 2011, AJ, 142, 152

Levison, H. F., Morbidelli, A., Vanlaerhoven, C., Gomes, R., \& Tsiganis, K. 2008, Icar, 196, 258

Lovis, C., Ségransan, D., Mayor, M., et al. 2011, A\&A, 528, A112

Mignard, F. 1979, M\&P, 20, 301

Minton, D. A., \& Malhotra, R. 2011, ApJ, 732, 53

Morbidelli, A., Brasser, R., Gomes, R., Levison, H. F., \& Tsiganis, K. 2010, AJ, 140,1391

Morbidelli, A., Levison, H. F., Tsiganis, K., \& Gomes, R. 2005, Natur, 435, 462

Morbidelli, A., Tsiganis, K., Crida, A., Levison, F. H., \& Gomes, R. 2007, AJ, 134,1790

Murray, C. D., \& Dermott, S. F. 1999, Solar System Dynamics (Cambridge: Cambridge Univ. Press)

Nesvorný, D. 2011, ApJ, 742, 22

Nesvorný, N., \& Morbidelli, A. 2012, AJ, 144, 117

Nesvorný, D., \& Vokrouhlický, D. 2009, AJ, 137, 5003

Nesvorný, D., Vokrouhlický, D., \& Deienno, R. 2014a, ApJ, 784, 22

Nesvorný, D., Vokrouhlický, D., Deienno, R., \& Walsh, K. J. 2014b, AJ, submitted

Nesvorný, D., Vokrouhlický, D., \& Morbidelli, A. 2007, AJ, 133, 1962

Nesvorný, D., Vokrouhlický, D., \& Morbidelli, A. 2013, ApJ, 768, 45

Tremaine, S., Touma, J., \& Namouni, F. 2009, AJ, 137, 3706

Tsiganis, K., Gomes, R. S., Morbidelli, A., \& Levison, H. F. 2005, Natur, 435,459

Van Laerhoven, C., \& Greenberg, R. 2013, ApJ, 778, 182

Ward, W. R., \& Canup, R. M. 2006, ApJL, 640, L91

Yoder, C. F., \& Peale, S. J. 1981, Icar, 47, 1 